\title{
The Intersection of Upper and Lower Semi-Browder Spectrum of Upper-Triangular Operator Matrices
}

\author{
Shifang Zhang, ${ }^{1}$ Huaijie Zhong, ${ }^{1}$ and Long Long ${ }^{2}$ \\ ${ }^{1}$ School of Mathematics and Computer Science, Fujian Normal University, Fuzhou 350007, China \\ ${ }^{2}$ School of Mathematics, Central South University, Changsha 410075, China \\ Correspondence should be addressed to Long Long; longwanglong@hotmail.com
}

Received 22 January 2013; Accepted 3 August 2013

Academic Editor: Alberto Fiorenza

Copyright (c) 2013 Shifang Zhang et al. This is an open access article distributed under the Creative Commons Attribution License, which permits unrestricted use, distribution, and reproduction in any medium, provided the original work is properly cited.

\begin{abstract}
When $A \in B(H)$ and $B \in B(K)$ are given, we denote by $M_{C}$ the operator acting on the infinite-dimensional separable Hilbert space $H \oplus K$ of the form $M_{C}=\left(\begin{array}{ll}A & C \\ 0 & B\end{array}\right)$. In this paper, it is proved that there exists some operator $C \in B(K, H)$ such that $M_{C}$ is upper semi-Browder if and only if there exists some left invertible operator $C \in B(K, H)$ such that $M_{C}$ is upper semi-Browder. Moreover, a necessary and sufficient condition for $M_{C}$ to be upper semi-Browder for some $C \in G(K, H)$ is given, where $G(K, H)$ denotes the subset of all of the invertible operators of $B(K, H)$.
\end{abstract}

\section{Introduction}

It is well known that if $H$ is a Hilbert space, $T$ is a bounded linear operator defined on $H$, and $H_{1}$ is an invariant closed subspace of $T$, then $T$ can be represented in the following form:

$$
T=\left(\begin{array}{cc}
* & * \\
0 & *
\end{array}\right): H_{1} \oplus H_{1}^{\perp} \longrightarrow H_{1} \oplus H_{1}^{\perp}
$$

which motivated the interest in $2 \times 2$ upper-triangular operator matrices. For recent investigations on this subject, see references [1-23].

Throughout this paper, let $H$ and $K$ be separable infinitedimensional complex Hilbert spaces, and let $B(H, K)$ be the set of all bounded linear operators from $H$ into $K$; when $H=K$, we write $B(H, H)$ as $B(H)$. For $A \in B(H), B \in B(K)$, and $C \in B(K, H)$, we have $M_{C}=\left(\begin{array}{ll}A & C \\ 0 & B\end{array}\right) \in B(H \oplus K)$. For $T \in B(H, K)$, let $R(T)$ and $N(T)$ denote the range and the kernel of $T$, respectively, and denote that $\alpha(T)=\operatorname{dim} N(T)$ and $\beta(T)=\operatorname{dim} K / R(T)$. If $T \in B(H)$, the ascent $\operatorname{asc}(T)$ of $T$ is defined to be the smallest nonnegative integer $k$ which satisfies and $N\left(T^{k}\right)=N\left(T^{k+1}\right)$. If such $k$ does not exist, then the ascent of $T$ is defined as infinity. Similarly, the descent $\operatorname{des}(T)$ of $T$ is defined as the smallest nonnegative integer $k$ for which $R\left(T^{k}\right)=R\left(T^{k+1}\right)$ holds. If such $k$ does not exist, then $\operatorname{des}(T)$ is defined as infinity, too. If the ascent and the descent of $T$ are finite, then they are equal (see [6]). For $T \in B(H)$, if $R(T)$ is closed and $\alpha(T)<\infty$, then $T$ is said to be an upper semi-Fredholm operator; if $\beta(T)<\infty$, which implies that $R(T)$ is closed, then $T$ is said to be a lower semiFredholm operator. If $T \in B(H)$ is either upper or lower semiFredholm operator, then $T$ is said to be a semi-Fredholm operator. If both $\alpha(T)<\infty$ and $\beta(T)<\infty$, then $T$ is said to be a Fredholm operator. For a semi-Fredholm operator $T$, its index ind $(T)$ is defined by ind $(T)=\alpha(T)-\beta(T)$.

For a semi-Fredholm operator $T \in B(H)$, its shift Samuel multiplicity $s \_m u l(T)$ and backward shift Samuel multiplicity b.s._mul( $T$ ) are defined, respectively, by the following (see [24]):

$$
\begin{gathered}
\text { s_mul }(T)=\lim _{k \rightarrow \infty} \frac{\beta\left(T^{k}\right)}{k}, \\
\text { b.s._mul }(T)=\lim _{k \rightarrow \infty} \frac{\alpha\left(T^{k}\right)}{k} .
\end{gathered}
$$

Moreover, it has been proved that $s_{-} \operatorname{mul}(T)$, b.s._mul $(T) \in$ $\{0,1,2, \ldots, \infty\}$ and that $\operatorname{ind}(T)=$ b.s._mul $(T)-s_{-} \operatorname{mul}(T)$. These two invariants refine the Fredholm index and can be regarded as the stabilized dimensions of the kernel and the cokernel (see [24]). 
In this paper, the sets of invertible operators and left invertible operators from $H$ into $K$ are denoted by $G(H, K)$ and $G_{l}(H, K)$, respectively; the sets of all Fredholm operators, upper semi-Fredholm operators, and lower semi-Fredholm operators from $H$ into $K$ are denoted by $\Phi(H, K), \Phi_{+}(H, K)$, and $\Phi_{-}(H, K)$, respectively; the sets of all Browder operators, upper semi-Browder operators, and lower semi-Browder operators, on $H$ are defined, respectively, by the following:

$$
\begin{gathered}
\Phi_{b}(H):=\{T \in \Phi(H): \operatorname{asc}(T)=\operatorname{des}(T)<\infty\}, \\
\Phi_{a b}(H):=\left\{T \in \Phi_{+}(H): \operatorname{asc}(T)<\infty\right\}, \\
\Phi_{s b}(H):=\left\{T \in \Phi_{-}(H): \operatorname{des}(T)<\infty\right\} .
\end{gathered}
$$

Moreover, for $T \in B(H)$, we introduce its corresponding spectra as follows.

The spectrum is given as $\sigma(T)=\{\lambda \in \mathbb{C}: T-\lambda I \notin$ $G(H)\}$.

The left spectrum is given as $\sigma_{l}(T)=\{\lambda \in \mathbb{C}: T-\lambda I \notin$ $\left.G_{l}(H)\right\}$.

The essential spectrum is defined as $\sigma_{e}(T)=\{\lambda \in \mathbb{C}$ : $T-\lambda I \notin \Phi(H)\}$.

The upper semi-Fredholm spectrum is defined as $\sigma_{S F+}(T)=\left\{\lambda \in \mathbb{C}: T-\lambda I \notin \Phi_{+}(X)\right\}$.

The lower semi-Fredholm spectrum is presented as $\sigma_{S F_{-}}(T)=\left\{\lambda \in \mathbb{C}: T-\lambda I \notin \Phi_{-}(X)\right\}$.

The Browder spectrum is presented as $\sigma_{b}(T)=\{\lambda \in$ $\left.\mathbb{C}: T-\lambda I \notin \Phi_{b}(H)\right\}$.

The upper semi-Browder spectrum is defined as $\sigma_{a b}(T)=\left\{\lambda \in \mathbb{C}: T-\lambda I \notin \Phi_{a b}(X)\right\}$.

The lower semi-Browder spectrum is presented as $\sigma_{s b}(T)=\left\{\lambda \in \mathbb{C}: T-\lambda I \notin \Phi_{s b}(X)\right\}$.

Using the Samuel multiplicities, Zhang and Wu (see [20]) gave a necessary and sufficient condition for which $M_{C} \in$ $\Phi_{a b}(H \oplus K)$ for some $C \in B(K, H)$ and characterized the set of $\cap_{C \in B(K, H)} \sigma_{a b}\left(M_{C}\right)$. In this paper, our main goal is to characterize the intersection of $\cap_{C \in G_{l}(K, H)} \sigma_{a b}\left(M_{C}\right)$ and $\cap_{C \in G(K, H)} \sigma_{a b}\left(M_{C}\right)$. This paper is organized as follows. In Section 2, we give a necessary and sufficient condition for which $M_{C} \in \Phi_{a b}(H \oplus K)$ for some $C \in G_{l}(K, H)$ and get

$$
\bigcap_{C \in G_{l}(K, H)} \sigma_{a b}\left(M_{C}\right)=\bigcap_{C \in B(K, H)} \sigma_{a b}\left(M_{C}\right) .
$$

In Section 3, we give a necessary and sufficient condition for which $M_{C} \in \Phi_{a b}(H \oplus K)$ for some $C \in G(K, H)$ and get

$$
\begin{aligned}
\bigcap_{C \in G(K, H)} \sigma_{a b}\left(M_{C}\right)= & \bigcap_{C \in \Phi(K, H)} \sigma_{a b}\left(M_{C}\right) \\
= & \left(\bigcap_{C \in B(K, H)} \sigma_{a b}\left(M_{C}\right)\right) \\
& \cup\{\lambda \in \mathbb{C}: B-\lambda \text { is compact }\} .
\end{aligned}
$$

For the sake of convenience, we now present some lemmas which will be used in the sequel.
Lemma 1 (see $[20,24]$ ). An operator $T \in B(H)$ is semiFredholm if and only if $T$ can be decomposed into the following form with respect to some orthogonal decomposition $H=H_{1} \oplus$ $\mathrm{H}_{2} \oplus \mathrm{H}_{3}$ :

$$
\begin{aligned}
T & =\left(\begin{array}{ccc}
T_{1} & T_{12} & T_{13} \\
0 & T_{2} & T_{23} \\
0 & 0 & T_{3}
\end{array}\right): H_{1} \oplus H_{2} \oplus H_{3} \\
& \longrightarrow H_{1} \oplus H_{2} \oplus H_{3},
\end{aligned}
$$

where $\operatorname{dim}\left(H_{3}\right)<\infty, T_{1}$ is a right invertible operator, $T_{3}$ is a finite nilpotent operator, $T_{2}$ is a left invertible operator, and $\min \left\{\right.$ ind $\left(T_{1}\right),-$ ind $\left.\left(T_{2}\right)\right\}<\infty$. Moreover, ind $\left(T_{1}\right)=$ $\alpha\left(T_{1}\right)=b . s$. $_{-} \operatorname{mul}(T)$, ind $\left(T_{2}\right)=-\beta\left(T_{2}\right)=-s_{-} \operatorname{mul}(T)$, and ind $(T)=\alpha\left(T_{1}\right)-\beta\left(T_{2}\right)$.

Lemma 2 (see [18]). Let $A \in B(H), B \in B(K)$, and $C \in$ $B(K, H)$.

(1) If $A \in \Phi_{b}(H)$, then $B \in \Phi_{a b}(K)$ if $M_{C} \in \Phi_{a b}(H \oplus K)$ for some $C \in B(K, H)$.

(2) If $M_{C} \in \Phi_{a b}(H \oplus K)$ for some $C \in B(K, H)$, then $A \in$ $\Phi_{a b}(H)$.

(3) If $A \in \Phi_{a b}(H)$ and $B \in \Phi_{a b}(K)$, then $M_{C} \in \Phi_{a b}(H \oplus$ $K)$ for any $C \in B(K, H)$.

(4) If $B \in \Phi_{b}(K)$, then $A \in \Phi_{a b}(H)$ if $M_{C} \in \Phi_{a b}(H \oplus K)$ for some $C \in B(K, H) ; A \in \Phi_{s b}(H)$ if $M_{C} \in \Phi_{s b}(H \oplus K)$ for some $C \in B(K, H)$.

(5) If $M_{C} \in \Phi_{b}(H \oplus K)$ for some $C \in B(K, H)$, then $A \in$ $\Phi_{a b}(H)$ and $B \in \Phi_{s b}(K)$.

(6) If two of $A, B$, and $M_{C}$ are Browder, then so is the third.

Lemma 3 (see [20]). Let $T \in B(H)$. Then, $T$ is upper semiBrowder if $T$ can be decomposed into the following form with respect to some orthogonal decomposition $\mathrm{H}=\mathrm{H}_{1} \oplus \mathrm{H}_{2}$ :

$$
T=\left(\begin{array}{cc}
T_{1} & T_{12} \\
0 & T_{2}
\end{array}\right),
$$

where $\operatorname{dim}\left(H_{1}\right)<\infty, T_{1}$ is nilpotent, $T_{2}$ is left invertible, and $\beta\left(T_{2}\right)=s_{-} \operatorname{mul}(T)=-\operatorname{ind}(T)$.

Lemma 4 (see [20]). Let $T \in B(H)$. Then, $T$ is lower semiBrowder if $T$ can be decomposed into the following form with respect to some orthogonal decomposition $\mathrm{H}=\mathrm{H}_{1} \oplus \mathrm{H}_{2}$ :

$$
T=\left(\begin{array}{cc}
T_{1} & T_{12} \\
0 & T_{2}
\end{array}\right)
$$

where $\operatorname{dim}\left(H_{2}\right)<\infty, T_{1}$ is right invertible, $T_{2}$ is nilpotent, and $\alpha\left(T_{1}\right)=$ b.s._ $\operatorname{mul}(T)=$ ind $(T)$.

Lemma 5 (see [20]). For any given $A \in B(H)$ and $B \in B(K)$, $M_{C} \in \Phi_{a b}(H \oplus K)$ for some $C \in B(K, H)$ if $A \in \Phi_{a b}(H)$ and

$$
\begin{aligned}
& s_{-} \operatorname{mul}(A)=\infty \quad \text { if } B \notin \Phi_{+}(K), \\
& \text { b.s._ mul }(B) \leq s_{-} \operatorname{mul}(A) \quad \text { if } B \in \Phi_{+}(K) .
\end{aligned}
$$


Lemma 6 (see [9]). For any given $A \in B(H)$ and $B \in B(K)$, $M_{C}$ is left invertible for some $C \in B(K, H)$ if $A$ is left invertible and

$$
\begin{aligned}
& a(B) \leq \beta(A) \quad \text { if } R(B) \text { is closed, } \\
& \beta(A)=\infty \quad \text { if } R(B) \text { is not closed. }
\end{aligned}
$$

Lemma 7 (see [25]). Let $V$ be a linear subspace of $H$. Then, the following statements are equivalent.

(1) Any bounded operator $A \in B(H)$ with $R(A) \subseteq V$ is compact.

(2) V contains no closed infinite-dimensional subspace.

\section{2. $\bigcap_{C \in B(K, H)} \sigma_{a b}\left(M_{C}\right)$ and $\bigcap_{C \in G_{l}(K, H)} \sigma_{a b}\left(M_{C}\right)$}

In $[1,20]$, the authors have proved that

$$
\begin{aligned}
\bigcap_{C \in B(K, H)} \sigma_{b}\left(M_{C}\right) & \\
=\sigma_{a b}(A) \cup \sigma_{s b}(B) \cup\{\lambda & \in \mathbb{C}: \alpha(A-\lambda) \\
& +\alpha(B-\lambda) \neq \beta(A-\lambda) \\
& +\beta(B-\lambda)\} .
\end{aligned}
$$

They, moreover, proved that

$$
\begin{aligned}
& \bigcap_{C \in B(K, H)} \sigma_{a b}\left(M_{C}\right) \\
& =\sigma_{a b}(A) \cup\left\{\lambda \in \mathbb{C}: \lambda \in \sigma_{S F+}(B),\right. \\
& \left.\quad s_{-} \operatorname{mul}(A-\lambda)<\infty\right\} \\
& \cup\left\{\lambda \in \Phi(A) \cap \Phi_{+}(B): \text { b.s._mul }(B-\lambda)\right. \\
& \left.>s_{-} \operatorname{mul}(A-\lambda)\right\} .
\end{aligned}
$$

Comparing the above two kinds of spectra with the upper semi-Weyl spectrum and Weyl spectrum, one may expect that the following equality holds:

$$
\begin{gathered}
\bigcap_{C \in B(K, H)} \sigma_{a b}\left(M_{C}\right) \\
=\sigma_{a b}(A) \cup\left\{\lambda \in \mathbb{C}: \lambda \in \sigma_{S F+}(B), \beta(A-\lambda)<\infty\right\} \\
\cup\{\lambda \in \mathbb{C}: \alpha(A-\lambda)+\alpha(B-\lambda) \\
>\beta(A-\lambda)+\beta(B-\lambda)\} .
\end{gathered}
$$

However, it is not that case, as the following example shows. Example 8. Let $A$ be the unilateral shift on $\ell^{2}$, that is,

$$
V: \ell^{2} \longrightarrow \ell^{2},\left\{z_{1}, z_{2}, \ldots\right\} \longmapsto\left\{0, z_{1}, z_{2}, \ldots\right\},
$$

and let the operators $A$ and $B$ be defined by

$$
A=V, \quad B=\left(\begin{array}{cc}
\left(V^{*}\right)^{2} & 0 \\
0 & V^{5}
\end{array}\right): \ell^{2} \oplus \ell^{2} \longrightarrow \ell^{2} \oplus \ell^{2} .
$$

Then, we have b.s. $\operatorname{mul}(B)=2>s \_\operatorname{mul}(A)=1$, while $\alpha(A)+\alpha(B)=2<\beta(A)+\beta(B)=6$. Moreover, $0 \epsilon$ $\bigcap_{C \in B(K, H)} \sigma_{a b}\left(M_{C}\right)$, while $0 \notin \sigma_{a b}(A) \cup\left\{\lambda \in \mathbb{C}: \lambda \in \sigma_{S F+}(B)\right.$ and $\beta(A-\lambda)<\infty\} \cup\{\lambda \in \mathbb{C}: \alpha(A-\lambda)+\alpha(B-\lambda)>$ $\beta(A-\lambda)+\beta(B-\lambda)\}$. Thus, (13) does not hold.

In spite of the above counter example, we have the following.

Proposition 9. For any given $A \in B(H)$ and $B \in B(K)$, one has

$$
\begin{aligned}
\bigcap_{C \in B(K, H)} \sigma_{a b}\left(M_{C}\right) \supseteq \sigma_{a b}(A) \\
\cup\left\{\lambda \in \mathbb{C}: \lambda \in \sigma_{S F+}(B), \beta(A-\lambda)<\infty\right\} \\
\cup\{\lambda \in \mathbb{C}: \alpha(A-\lambda)+\alpha(B-\lambda) \\
>\beta(A-\lambda)+\beta(B-\lambda)\} .
\end{aligned}
$$

Proof. From the proof of Theorem 2.3 in [20], we know that when $A \in \Phi_{a b}(H), s_{-} \operatorname{mul}(A)<\infty$ if and only if $\beta(A)<\infty$. Combining this fact with Corollary 2.5 of [20], it is easy to see that

$$
\bigcap_{C \in B(K, H)} \sigma_{a b}\left(M_{C}\right) \supseteq \sigma_{a b}(A) \cup\left\{\lambda \in \mathbb{C}: \lambda \in \sigma_{S F+}(B),\right.
$$

$$
\beta(A-\lambda)<\infty\}
$$

Noting that $\beta(B-\lambda)<\infty$ implies that $R(B-\lambda)$ is closed, it follows from corollary 2.5 of [2] that

$$
\begin{aligned}
\bigcap_{C \in B(K, H)} \sigma_{a b}\left(M_{C}\right) \supseteq \bigcap_{C \in B(K, H)} \sigma_{a w}\left(M_{C}\right) \\
\supseteq\{\lambda \in \mathbb{C}: \alpha(A-\lambda)+\alpha(B-\lambda) \\
\\
\quad>\beta(A-\lambda)+\beta(B-\lambda)\},
\end{aligned}
$$

where $\sigma_{a w}\left(M_{C}\right)=\left\{\lambda \in \mathbb{C}: M_{C}-\lambda\right.$ is not uppersemiFredholm operator with index less than or equal to 0$\}$.

Now, we are ready to present the main result of this section.

Theorem 10. For any given $A \in B(H)$ and $B \in B(K)$, one has

$$
\bigcap_{C \in G_{l}(K, H)} \sigma_{a b}\left(M_{C}\right)=\bigcap_{C \in B(K, H)} \sigma_{a b}\left(M_{C}\right) .
$$

Proof. Since $\bigcap_{C \in G_{l}(K, H)} \sigma_{a b}\left(M_{C}\right) \supseteq \bigcap_{C \in B(K, H)} \sigma_{a b}\left(M_{C}\right)$ is obvious, it is sufficient to prove that if $M_{C} \in \Phi_{a b}(H \oplus K)$, then there exists some left invertible operator $Q \in B(K, H)$ such that $M_{Q} \in \Phi_{a b}(H \oplus K)$.

Suppose that $M_{C} \in \Phi_{a b}(H \oplus K)$. It follows from Lemma 5 that $A \in \Phi_{a b}(H)$ and

$$
\begin{aligned}
& s_{-} \operatorname{mul}(A)=\infty \quad \text { if } B \notin \Phi_{+}(K), \\
& \text { b.s._mul }(B) \leq s_{-} \operatorname{mul}(A) \quad \text { if } B \in \Phi_{+}(K) .
\end{aligned}
$$

There are two cases to consider. 
Case 1. Assume that $A \in \Phi_{a b}(H), s_{-} \operatorname{mul}(A)=\infty$, and $B \notin \Phi_{+}(K)$. Then, it follows from Lemma 3 that $A$ can be decomposed into the following form:

$$
A=\left(\begin{array}{cc}
A_{1} & A_{12} \\
0 & A_{2}
\end{array}\right): H_{1} \oplus H_{2} \longrightarrow H_{1} \oplus H_{2}
$$

where $\operatorname{dim}\left(H_{1}\right)<\infty, A_{1}$ is nilpotent, $A_{2}$ is a left invertible operator, and $\beta\left(A_{2}\right)=s \_$mul $(A)=\infty$. So, we can let

$$
Q=\left(\begin{array}{c}
0 \\
0 \\
V
\end{array}\right): K \longrightarrow H_{1} \oplus R\left(A_{2}\right) \oplus\left(H_{2} \ominus R\left(A_{2}\right)\right),
$$

where $V \in B\left(K,\left(H_{2} \ominus R\left(A_{2}\right)\right)\right)$ is unitary. Obviously, $Q$ is left invertible. Now, $M_{\mathrm{Q}}$ can be rewritten as

$$
\begin{aligned}
M_{Q} & =\left(\begin{array}{ccc}
A_{1} & A_{12} & 0 \\
0 & A_{2} & 0 \\
0 & 0 & V \\
0 & 0 & B
\end{array}\right): H_{1} \oplus H_{2} \oplus K \\
& \longrightarrow H_{1} \oplus R\left(A_{2}\right) \oplus\left(H_{2} \ominus R\left(A_{2}\right)\right) \oplus K .
\end{aligned}
$$

Since $A_{2}$ is left invertible and $V$ is invertible, then there exist unique $A_{2}^{\prime}$ and $V^{\prime}$ such that $A_{2}^{\prime} A_{2}=I_{H_{2}}$ and $V^{\prime} V=I_{K}$, and

$$
\left(\begin{array}{ccc}
A_{2}^{\prime} & 0 & 0 \\
0 & V^{\prime} & 0
\end{array}\right)\left(\begin{array}{cc}
A_{2} & 0 \\
0 & V \\
0 & B
\end{array}\right)=I_{H_{2}} \oplus I_{K}
$$

This implies that $\left(\begin{array}{cc}A_{2} & 0 \\ 0 & V \\ 0 & B\end{array}\right)$ is left invertible. And, hence, Lemma 2 leads to $M_{\mathrm{Q}} \in \Phi_{a b}(H \oplus K)$.

Case 2. Assume that $A \in \Phi_{a b}(H)$, b.s._mul( $\left.B\right) \leq s_{-} \operatorname{mul}(A)$, and $B \in \Phi_{+}(K)$. Then, it follows from Lemma 3 that $A$ can be decomposed into the following form:

$$
A=\left(\begin{array}{cc}
A_{1} & A_{12} \\
0 & A_{2}
\end{array}\right): H_{1} \oplus H_{2} \longrightarrow H_{1} \oplus H_{2},
$$

where $\operatorname{dim}\left(H_{1}\right)<\infty, A_{1}$ is nilpotent, $A_{2}$ is a left invertible operator, and $\beta\left(A_{2}\right)=s_{-} \operatorname{mul}(A)$. By the assumption that $B \in \Phi_{+}(K)$ and Lemma 1 , we know that $B$ can be decomposed into the following form with respect to some orthogonal decomposition $K=K_{1} \oplus K_{2} \oplus K_{3}$ :

$$
B=\left(\begin{array}{ccc}
B_{1} & * & * \\
0 & B_{2} & * \\
0 & 0 & B_{3}
\end{array}\right)
$$

where $\operatorname{dim}\left(K_{3}\right)<\infty, B_{1}$ is a right invertible operator, $B_{2}$ is a left invertible operator, $B_{3}$ is a finite nilpotent operator, and the parts marked by $*$ can be any operators. Moreover, $\infty>$ $\alpha\left(B_{1}\right)=$ b.s. $\operatorname{mul}(B)$. Thus, $\beta\left(A_{2}\right) \geq \alpha\left(B_{1}\right)$, and then there exists some left invertible $C_{1} \in B\left(N\left(B_{1}\right), H_{2} \ominus R\left(A_{2}\right)\right)$. Noting that $\operatorname{dim}\left(\left(K_{1} \ominus N\left(B_{1}\right)\right) \oplus K_{2} \oplus K_{3}\right)=\operatorname{dim}\left(H_{1} \oplus R\left(A_{2}\right)\right)=\infty$, we can let $C_{2} \in G\left(\left(K_{1} \ominus N\left(B_{1}\right)\right) \oplus K_{2} \oplus K_{3}, H_{1} \oplus R(A)\right)$. Consider

$$
\begin{aligned}
Q & =\left(\begin{array}{cc}
C_{1} & 0 \\
0 & C_{2}
\end{array}\right): N\left(B_{1}\right) \oplus\left[K_{1} \ominus N\left(B_{1}\right) \oplus K_{2} \oplus K_{3}\right] \\
& \longrightarrow\left(H_{2} \ominus R\left(A_{2}\right)\right) \oplus\left[H_{1} \oplus R\left(A_{2}\right)\right] .
\end{aligned}
$$

Obviously, $Q$ is left invertible, and $M_{\mathrm{Q}}$ can be rewritten as

$$
\begin{aligned}
M_{Q} & \left(\begin{array}{cccccc}
A_{1} & A_{12} & C_{11} & 0 & C_{12} & C_{13} \\
0 & A_{21} & C_{21} & 0 & C_{22} & C_{23} \\
0 & 0 & 0 & C_{1} & 0 & 0 \\
0 & 0 & B_{11} & 0 & * & * \\
0 & 0 & 0 & 0 & B_{2} & * \\
0 & 0 & 0 & 0 & 0 & B_{3}
\end{array}\right): H_{1} \oplus H_{2} \\
& \oplus\left(K_{1} \ominus N\left(B_{1}\right) \oplus N\left(B_{1}\right)\right) \\
& \oplus K_{2} \oplus K_{3} \\
\longrightarrow & H_{1} \oplus R\left(A_{2}\right) \oplus\left(H_{2} \ominus d R\left(A_{2}\right)\right) \oplus K_{1} \\
& \oplus K_{2} \oplus K_{3},
\end{aligned}
$$

where $A_{21}$ and $B_{11}$ are invertible and $C_{1}$ and $B_{2}$ are left invertible. Similar to the proof of Case 1, through direct calculation we can show that $\left(\begin{array}{ccc}A_{21} & C_{21} & 0 \\ 0 & 0 & C_{1} \\ 0 & B_{11} & 0\end{array}\right)$ is left invertible. Also since $\operatorname{dim}\left(H_{1}\right)<\infty$ and $\operatorname{dim}\left(K_{3}\right)<\infty$, we have $A_{1} \epsilon$ $\Phi_{b}\left(H_{1}\right)$ and $B_{3} \in \Phi_{b}\left(K_{3}\right)$. Thus, it follows from Lemma 2 that $M_{C} \in \Phi_{a b}(H \oplus K)$.

By duality, we have the following.

Theorem 11. For any given $A \in B(H)$ and $B \in B(K)$, one has

$$
\bigcap_{C \in G_{r}(K, H)} \sigma_{s b}\left(M_{C}\right)=\bigcap_{C \in B(K, H)} \sigma_{s b}\left(M_{C}\right) .
$$

\section{3. $\bigcap_{C \in G(K, H)} \sigma_{a b}\left(M_{C}\right)$ and $\bigcap_{C \in \Phi(K, H)} \sigma_{a b}\left(M_{C}\right)$}

In this section, we give the characterization of invertible and Fredholm perturbations of upper semi-Browder spectra of $2 \times$ 2 upper-triangular matrices. We begin with some lemmas.

Lemma 12 (see [19]). For a given pair $(A, B) \in B(H) \times B(K)$, if either $A$ or $B$ is a compact operator, then, for each $C \in \Phi(K, H)$, $M_{C}$ is not a semi-Fredholm operator.

In particular, if $B$ is not compact, then $M_{C}$ is not semiBrowder for any invertible operator $C$.

Lemma 13. The following statements are equivalent.

(i) $B$ is not compact.

(ii) For each given $A \in \Phi_{a b}(H)$, if $\beta(A)=\infty$, then there exists an operator $C \in G(K, H)$ such that $M_{C}$ is an upper semi-Browder operator.

(iii) For each given $A \in \Phi_{a b}(H)$, if $\beta(A)=\infty$, then there exists an operator $C \in \Phi(K, H)$ such that $M_{C}$ is an upper semi-Browder operator.

Proof. Obviously, we only need to prove the implications (i) $\Rightarrow$ (ii) and (iii) $\Rightarrow$ (i).

(iii) $\Rightarrow$ (i). If $B$ is compact, then it follows from Lemma 12 that $M_{C}$ is not a semi-Fredholm operator for each $C \in \Phi(K, H)$, which contradicts with (iii). Thus, $B$ is not compact. 
(i) $\Rightarrow$ (ii). Suppose that $B$ is not compact. Then, we consider the following two cases.

Case 1. Assume that $R(B)$ is closed. It follows from Lemma 3 that $A$ can be decomposed into the following form with respect to some orthogonal decomposition $H=H_{1} \oplus H_{2}$ :

$$
A=\left(\begin{array}{cc}
A_{1} & A_{12} \\
0 & A_{2}
\end{array}\right): H_{1} \oplus H_{2} \longrightarrow H_{1} \oplus H_{2}
$$

where $\operatorname{dim}\left(H_{1}\right)<\infty, A_{1}$ is nilpotent, and $A_{2}$ is a left invertible operator. Noting that $\beta(A)=\infty$, we have $\beta\left(A_{2}\right)=$ $\infty$. Since the assumption that $B$ is not compact, we have that $\operatorname{dim} N(B)^{\perp}=\infty$. Also since $\beta\left(A_{2}\right)=\infty$, let $R\left(A_{2}\right)^{\perp}=$ $H_{3} \oplus H_{4}$ with $\operatorname{dim}\left(H_{3}\right)=\operatorname{dim} N(B)$ and $\operatorname{dim}\left(H_{4}\right)=\infty$. Define an operator $C: K \rightarrow H$ by

$$
\begin{aligned}
C & =\left(\begin{array}{cc}
C_{1} & 0 \\
0 & C_{2}
\end{array}\right): N(B) \oplus N(B)^{\perp} \\
& \longrightarrow H_{3} \oplus\left(H_{1} \oplus R\left(A_{2}\right) \oplus H_{4}\right),
\end{aligned}
$$

where $C_{1} \in B\left(N(B), H_{3}\right)$ and $C_{2} \in B\left(N(B)^{\perp}, H_{1} \oplus R\left(A_{2}\right) \oplus H_{4}\right)$ are invertible operators. Obviously, $C \in B(K, H)$ is invertible. Next, we claim that $M_{C}$ is an upper semi-Browder operator. To see this, $M_{C}$ can be rewritten as

$$
\begin{aligned}
M_{C}= & \left(\begin{array}{cccc}
A_{1} & A_{12} & C_{11} & 0 \\
0 & A_{22} & C_{21} & 0 \\
0 & 0 & 0 & C_{1} \\
0 & 0 & C_{41} & 0 \\
0 & 0 & B_{1} & 0
\end{array}\right): H_{1} \oplus H_{2} \\
& \oplus N(B)^{\perp} \oplus N(B) \\
& \longrightarrow H_{1} \oplus R\left(A_{2}\right) \oplus H_{3} \oplus H_{4} \oplus K,
\end{aligned}
$$

where $A_{22} \in B\left(H_{2}, R\left(A_{2}\right)\right)$ is invertible and $B_{1} \in$ $B\left(N(B)^{\perp}, R(B)\right)$ is left invertible. By Lemma 2 and the fact that $A_{1} \in \Phi_{b}\left(H_{1}\right)$, it is sufficient to prove that

$$
M_{1}=:\left(\begin{array}{ccc}
A_{22} & C_{21} & 0 \\
0 & 0 & C_{1} \\
0 & C_{41} & 0 \\
0 & B_{1} & 0
\end{array}\right)
$$

is semi-Browder. For this, we only need to show that $M_{1}$ is left invertible. In fact, since $A_{22}$ is invertible and $B_{1}$ and $C_{1}$ are left invertible, we can set $A_{22}^{\prime}, B_{1}^{\prime}$, and $C_{1}^{\prime}$ such that

$$
A_{22}^{\prime} A_{22}=I_{H_{2}}, \quad B_{1}^{\prime} B_{1}=I_{N(B)^{\perp}}, \quad C_{1}^{\prime} C_{1}=I_{N(B)} .
$$

Direct calculation shows that

$$
\begin{gathered}
\left(\begin{array}{cccc}
A_{22}^{\prime} & 0 & 0 & -A_{22}^{\prime} C_{21} B_{1}^{\prime} \\
0 & 0 & 0 & B_{1}^{\prime} \\
0 & C_{1}^{\prime} & 0 & 0
\end{array}\right)\left(\begin{array}{ccc}
A_{22} & C_{21} & 0 \\
0 & 0 & C_{1} \\
0 & C_{41} & 0 \\
0 & B_{1} & 0
\end{array}\right) \\
=\left(\begin{array}{ccc}
I_{H_{2}} & 0 & 0 \\
0 & I_{N(B)^{\perp}} & 0 \\
0 & 0 & I_{N(B)}
\end{array}\right),
\end{gathered}
$$

which implies that $M_{1}$ is left invertible. Noting that $A_{1} \in$ $\Phi\left(H_{1}\right)$, by Lemma 2 we have that $M_{C}$ is upper semi-Browder.

Case 2. Assume that $R(B)$ is not closed. If $B$ is not compact, then by Lemma 7, $R(B)$ contains a closed infinitedimensional subspace. Without loss of generality, suppose that $\widetilde{K_{1}}$ is a closed subspace of $R(B)$ with $\operatorname{dim} \widetilde{K_{1}}=\infty$ and $\operatorname{dim}{\widetilde{K_{1}}}^{\perp}=\infty$. Let $K_{1}=\left\{x \in N(B)^{\perp}: B x \in \widetilde{K_{1}}\right\}$. Thus, $K_{1}$ is a closed subspace of $N(B)^{\perp}$, and $\operatorname{dim}\left(K_{1}\right)=\infty$. Denote $K_{2}=N(B)^{\perp} \ominus K_{1}$. Without loss of generality, we may assume that $\operatorname{dim}\left(K_{2}\right)=\infty$ (otherwise, suppose that $\left\{e_{n}\right\}_{n=1}^{\infty}$ is an orthonormal basis of $K_{1}$. Denote $K_{1}^{\prime}=\operatorname{span}\left\{e_{n}\right.$ : $n=2,4,6, \ldots\}$ and $\widetilde{K_{1}^{\prime}}=\left\{B x: x \in K_{1}^{\prime}\right\}$, then $K_{1}$ and $\widetilde{K_{1}}$ can be replaced by $K_{1}^{\prime}$ and $\widetilde{K_{1}^{\prime}}$, resp.). Since $\beta\left(A_{2}\right)=\infty$, let $R\left(A_{2}\right)^{\perp}=H_{3} \oplus H_{4}$ with $\operatorname{dim}\left(H_{3}\right)=\operatorname{dim} N(B)$ and $\operatorname{dim} H_{4}=\infty$. Define an operator $C: K \rightarrow H$ by

$$
\begin{aligned}
C & =\left(\begin{array}{ccc}
C_{1} & 0 & 0 \\
0 & C_{2} & 0 \\
0 & 0 & C_{3}
\end{array}\right): K_{1} \oplus K_{2} \oplus N(B) \\
& \longrightarrow\left(H_{1} \oplus R\left(A_{2}\right)\right) \oplus H_{4} \oplus H_{3},
\end{aligned}
$$

where $C_{1}, C_{2}$, and $C_{3}$ are unitary operators. Obviously, $C$ is invertible. $M_{C}$ can be rewritten as

$$
\begin{aligned}
M_{C}= & \left(\begin{array}{ccccc}
A_{1} & A_{12} & C_{11} & 0 & 0 \\
0 & A_{22} & C_{21} & 0 & 0 \\
0 & 0 & 0 & 0 & C_{3} \\
0 & 0 & 0 & C_{2} & 0 \\
0 & 0 & B_{11} & B_{12} & 0 \\
0 & 0 & 0 & B_{22} & 0
\end{array}\right): H_{1} \oplus H_{2} \\
& \oplus K_{1} \oplus K_{2} \oplus N(B) \\
& \longrightarrow H_{1} \oplus R\left(A_{2}\right) \oplus H_{3} \oplus H_{4} \oplus \widetilde{K_{1}} \oplus{\widetilde{K_{1}}}^{\perp},
\end{aligned}
$$

where $A_{22}$ and $B_{11}$ are invertible and $C_{1}=\left(\begin{array}{l}C_{11} \\ C_{21}\end{array}\right)$.

Next, we prove that $M_{C} \in \Phi_{a b}(H \oplus K)$. Noting that $\operatorname{dim}\left(H_{1}\right)<\infty$, then, by Lemma 2 , it is sufficient to prove that

$$
M_{1}=:\left(\begin{array}{cccc}
A_{22} & C_{21} & 0 & 0 \\
0 & 0 & 0 & C_{3} \\
0 & 0 & C_{2} & 0 \\
0 & B_{11} & B_{12} & 0 \\
0 & 0 & B_{22} & 0
\end{array}\right)
$$

is left invertible. For this, let $A_{22}^{\prime}, B_{11}^{\prime}, C_{1}^{\prime}$, and $C_{2}^{\prime}$ be operators satisfying

$$
\begin{aligned}
& A_{22}^{\prime} A_{22}=I_{H_{2}}, \quad B_{11}^{\prime} B_{11}=I_{K_{1}}, \\
& C_{2}^{\prime} C_{2}=I_{K_{2}}, \quad C_{3}^{\prime} C_{3}=I_{N(B)} .
\end{aligned}
$$


Direct calculation shows that

$$
\begin{aligned}
\left(\begin{array}{ccccc}
A_{22}^{\prime} & 0 & A_{22}^{\prime} C_{21} B_{11}^{\prime} B_{12} C_{2}^{\prime} & -A_{22}^{\prime} C_{21} B_{11}^{\prime} & 0 \\
0 & 0 & B_{11}^{\prime} B_{12} C_{2}^{\prime} & B_{11}^{\prime} & 0 \\
0 & 0 & C_{2}^{\prime} & 0 & 0 \\
0 & C_{3}^{\prime} & 0 & 0 &
\end{array}\right) \\
\quad\left(\begin{array}{cccc}
A_{22} & C_{21} & 0 & 0 \\
0 & 0 & 0 & C_{3} \\
0 & 0 & C_{2} & 0 \\
0 & B_{11} & B_{12} & 0 \\
0 & 0 & B_{22} & 0
\end{array}\right) \\
=\left(\begin{array}{cccc}
I_{H_{2}} & 0 & 0 & 0 \\
0 & I_{K_{1}} & 0 & 0 \\
0 & 0 & I_{K_{2}} & 0 \\
0 & 0 & 0 & I_{N(B)}
\end{array}\right),
\end{aligned}
$$

which implies that $M_{1}$ is left invertible.

Combining Case 1 with Case 2, the lemma is proved.

Similarly, we have the following.

Lemma 14. The following statements are equivalent:

(i) $A$ is not compact.

(ii) For each given $B \in \Phi_{s b}(H)$, if $\alpha(B)=\infty$, then there exists an operator $C \in G(K, H)$ such that $M_{C}$ is a lower semi-Browder operator.

(iii) For each given $B \in \Phi_{s b}(H)$, if $\alpha(B)=\infty$, then there exists an operator $C \in \Phi(K, H)$ such that $M_{C}$ is a lower semi-Browder operator.

One is now ready to prove the main result of this section.

Theorem 15. For a given pair $(A, B) \in B(H) \times B(K)$, one has

$$
\begin{aligned}
\bigcap_{C \in G(K, H)} \sigma_{a b}\left(M_{C}\right)= & \bigcap_{C \in \Phi(K, H)} \sigma_{a b}\left(M_{C}\right) \\
= & \left(\bigcap_{C \in B(K, H)} \sigma_{a b}\left(M_{C}\right)\right) \\
& \cup\{\lambda \in \mathbb{C}: B-\lambda \text { is compact }\} .
\end{aligned}
$$

Proof. According to Lemma 12, it is clear that

$$
\begin{aligned}
\bigcap_{C \in G(K, H)} \sigma_{a b}\left(M_{C}\right) \supseteq \bigcap_{C \in \Phi(K, H)} \sigma_{a b}\left(M_{C}\right) \\
\left.\supseteq \bigcap_{C \in B(K, H)} \sigma_{a b}\left(M_{C}\right)\right) \\
\\
\cup\{\lambda \in \mathbb{C}: B-\lambda \text { is compact }\} .
\end{aligned}
$$

For the conversion, without loss of generality, suppose that

$$
0 \notin\left(\bigcap_{C \in B(K, H)} \sigma_{a b}\left(M_{C}\right)\right) \cup\{\lambda \in \mathbb{C}: B-\lambda \text { is compact }\} .
$$

Then, $B$ is not compact, and there exists some $C \in$ $B(K, H)$ such that $M_{C} \in \Phi_{a b}(H \oplus K)$, and, hence, $A \in \Phi_{a b}(H)$.

Case 1. $\beta(A)=\infty$. It follows from Lemma 13 that there exists some $C \in G(K, H)$ such that $M_{C}$ is an upper semi-Browder operator. This implies that $\lambda \notin \bigcap_{C \in G(K, H)} \sigma_{a b}\left(M_{C}\right)$. In this case, we have proved. Consider that

$$
\begin{aligned}
\bigcap_{C \in G(K, H)} \sigma_{a b}\left(M_{C}\right) \subseteq & \left(\bigcap_{C \in B(K, H)} \sigma_{a b}\left(M_{C}\right)\right) \\
& \cup\{\lambda \in \mathbb{C}: B-\lambda \text { is compact }\} .
\end{aligned}
$$

Case 2. Consider $\beta(A)<\infty$. This implies that $A \in \Phi(H)$, and, thus, $B \in \Phi_{+}(K)$ since $M_{C} \in \Phi_{a b}(H \oplus K)$. It follows from Lemma 5 that $b . s . \_m u l(B) \leq s_{\_} \operatorname{mul}(A)$. Moreover, using Lemmas 1 and 3, we have

$$
\begin{gathered}
A=\left(\begin{array}{cc}
A_{1} & A_{12} \\
0 & A_{2}
\end{array}\right): H_{1} \oplus H_{2} \longmapsto H_{1} \oplus H_{2}, \\
B=\left(\begin{array}{ccc}
B_{1} & * & * \\
0 & B_{2} & * \\
0 & 0 & B_{3}
\end{array}\right): K_{1} \oplus K_{2} \oplus K_{3} \longmapsto K_{1} \oplus K_{2} \oplus K_{3},
\end{gathered}
$$

where $\operatorname{dim}\left(H_{1}\right)<\infty, A_{1}$ is nilpotent, $A_{2}$ is a left invertible operator, $\operatorname{dim}\left(K_{3}\right)<\infty, B_{1}$ is a right invertible operator, $B_{2}$ is a left invertible operator, $B_{3}$ is a finite nilpotent operator, and the parts marked by $*$ can be any operators. Moreover, $\beta\left(A_{2}\right)=s_{-} \operatorname{mul}(A)$, and $\alpha\left(B_{1}\right)=$ b.s._mul $(B)$. Hence, $\alpha\left(B_{1}\right) \leq$ $\beta\left(A_{2}\right)$. Now, put $H_{2}=R\left(A_{2}\right) \oplus H_{3} \oplus H_{4}$, where $\operatorname{dim}\left(H_{3}\right)=$ $\alpha\left(B_{1}\right)<\infty$. Noting that $\operatorname{dim} H_{1} \oplus R\left(A_{2}\right) \oplus H_{4}=N\left(B_{1}\right)^{\perp} \oplus$ $K_{2} \oplus K_{3}=\infty$, there exist unitaries $C_{33} \in B\left(N\left(B_{1}\right), H_{3}\right)$ and $C^{\prime} \in B\left(H_{1} \oplus R\left(A_{2}\right) \oplus H_{4}, N\left(B_{1}\right)^{\perp} \oplus K_{2} \oplus K_{3}\right)$. Let $C=\left(\begin{array}{cc}C_{33} & 0 \\ 0 & C^{\prime}\end{array}\right)$. Obviously, $C=\left(\begin{array}{cc}C_{33} & 0 \\ 0 & C^{\prime}\end{array}\right) \in G(H \oplus K)$.

Consider that operator

$M_{C}$

$$
\begin{aligned}
&=\left(\begin{array}{cc}
A & C \\
0 & B
\end{array}\right): H \oplus K \rightarrow H \oplus K \\
&\left(\begin{array}{cccccc}
A_{1} & A_{12} & C_{11} & 0 & C_{13} & C_{14} \\
0 & A_{2} & C_{21} & 0 & C_{23} & C_{24} \\
0 & 0 & 0 & C_{33} & 0 & 0 \\
0 & 0 & C_{41} & 0 & C_{43} & C_{44} \\
0 & 0 & B_{11} & 0 & * & * \\
0 & 0 & 0 & 0 & B_{2} & * \\
0 & 0 & 0 & 0 & 0 & B_{3}
\end{array}\right): H_{1} \oplus H_{2} \\
& \oplus N\left(B_{1}\right)^{\perp} \oplus N\left(B_{1}\right) \oplus K_{2} \oplus K_{3} \\
& \longrightarrow H_{1} \oplus R\left(A_{2}\right) \oplus H_{3} \oplus H_{4} \oplus K_{1} \oplus K_{2} \oplus K_{3},
\end{aligned}
$$

where

$$
\begin{aligned}
C^{\prime} & =\left(\begin{array}{lll}
C_{11} & C_{13} & C_{14} \\
C_{21} & C_{23} & C_{24} \\
C_{41} & C_{43} & C_{44}
\end{array}\right): H_{1} \oplus H_{2} \oplus N\left(B_{1}\right)^{\perp} \\
& \longrightarrow H_{1} \oplus R\left(A_{2}\right) \oplus H_{4}, \quad B_{1}=\left(\begin{array}{c}
B_{11} \\
0
\end{array}\right) .
\end{aligned}
$$


We claim that $M_{C} \in \Phi_{a b}(H \oplus K)$. In fact, since $A_{1}$ and $B_{3}$ are Browder operators, then, by Lemma 2, it is sufficient to show that

$$
\begin{aligned}
M= & :\left(\begin{array}{cccc}
A_{2} & C_{21} & 0 & C_{23} \\
0 & 0 & C_{33} & 0 \\
0 & C_{41} & 0 & C_{43} \\
0 & B_{11} & 0 & * \\
0 & 0 & 0 & B_{2}
\end{array}\right): H_{2} \oplus N\left(B_{1}\right)^{\perp} \\
& \oplus N\left(B_{1}\right) \oplus K_{2} \\
& \longrightarrow R\left(A_{2}\right) \oplus H_{3} \oplus H_{4} \oplus K_{1} \oplus K_{2}
\end{aligned}
$$

is upper semi-Browder. Observe that $A_{2}$ and $B_{2}$ are left invertible; $C_{33}$ and $B_{11}$ are invertible. Direct calculation shows that $M$ is injective. Since $A \in \Phi(H)$ and $B \in \Phi_{+}(K)$, we have $M_{C} \in \Phi_{a b}(H \oplus K)$, and, hence, $M$ is an upper semiFredholm operator. Thus, $M$ is left invertible. Combining this with Lemma 2 yields $M_{C} \in \Phi_{a b}(H \oplus K)$, which means that $\lambda \notin \bigcap_{C \in G(K, H)} \sigma_{a b}\left(M_{C}\right)$. Thus,

$$
\begin{aligned}
\bigcap_{C \in G(K, H)} \sigma_{a b}\left(M_{C}\right) \subseteq & \bigcap_{C \in B(K, H)} \sigma_{a b}\left(M_{C}\right) \\
& \cup\{\lambda \in \mathbb{C}: B-\lambda \text { is compact }\} .
\end{aligned}
$$

Combining Case 1 with Case 2 leads to

$$
\begin{aligned}
\bigcap_{C \in G(K, H)} \sigma_{a b}\left(M_{C}\right)= & \bigcap_{C \in \phi(K, H)} \sigma_{a b}\left(M_{C}\right) \\
= & \left(\bigcap_{C \in B(K, H)} \sigma_{a b}\left(M_{C}\right)\right) \\
& \cup\{\lambda \in \mathbb{C}: B-\lambda \text { is compact }\} .
\end{aligned}
$$

This completes the proof.

By duality, we have

Theorem 16. For a given pair $(A, B) \in B(H) \times B(K)$, one has

$$
\begin{aligned}
\bigcap_{C \in G(K, H)} \sigma_{s b}\left(M_{C}\right)= & \bigcap_{C \in \Phi(K, H)} \sigma_{s b}\left(M_{C}\right) \\
= & \left(\bigcap_{C \in B(K, H)} \sigma_{s b}\left(M_{C}\right)\right) \\
& \cup\{\lambda \in \mathbb{C}: A-\lambda \text { is compact }\} .
\end{aligned}
$$

\section{Acknowledgments}

Long Long research is supported by the Freedom Explore Program of Central South University (Grant no. 2012QNZT040) and the Post Doctoral Program Foundation of Central South University. This work is also supported by the National Natural Science Foundation of China (11226113, 11301077, 11171066, and 10771191), the Foundation of the Education Department of Fujian Province (JA12074), and the Natural Science Foundation of Fujian Province (2012J05003).

\section{References}

[1] X. H. Cao, "Browder spectra for upper triangular operator matrices," Journal of Mathematical Analysis and Applications, vol. 342, no. 1, pp. 477-484, 2008.

[2] X. H. Cao and B. Meng, "Essential approximate point spectra and Weyl's theorem for operator matrices," Journal of Mathematical Analysis and Applications, vol. 304, no. 2, pp. 759-771, 2005.

[3] X. L. Chen, S. F. Zhang, and H. J. Zhong, "On the filling in holes problem for operator matrices," Linear Algebra and its Applications, vol. 430, no. 1, pp. 558-563, 2009.

[4] D. S. Djordjević, "Perturbations of spectra of operator matrices," Journal of Operator Theory, vol. 48, no. 3, pp. 467-486, 2002.

[5] H. K. Du and P. Jin, "Perturbation of spectrums of $2 \times 2$ operator matrices," Proceedings of the American Mathematical Society, vol. 121, no. 3, pp. 761-766, 1994.

[6] S. V. Djordjević and Y. M. Han, "Spectral continuity for operator matrices," Glasgow Mathematical Journal, vol. 43, no. 3, pp. 487490, 2001.

[7] G. J. Hai and A. Chen, "Perturbations of the right and left spectra for operator matrices," Journal of Operator Theory, vol. 67, no. 1, pp. 207-214, 2012.

[8] J. K. Han, H. Y. Lee Youl, and W. Y. Lee Young, "Invertible completions of $2 \times 2$ upper triangular operator matrices," Proceedings of the American Mathematical Society, vol. 128, no. 1, pp. 119-123, 2000.

[9] I. S. Hwang and W. Y. Lee, "The boundedness below of $2 \times$ 2 upper triangular operator matrices," Integral Equations and Operator Theory, vol. 39, no. 3, pp. 267-276, 2001.

[10] W. Y. Lee, "Weyl's theorem for operator matrices," Integral Equations and Operator Theory, vol. 32, no. 3, pp. 319-331, 1998.

[11] W. Y. Lee, "Weyl spectra of operator matrices," Proceedings of the American Mathematical Society, vol. 129, no. 1, pp. 131-138, 2001.

[12] M. Z. Kolundžija and D. S. Djordjević, "Generalized invertibility of operator matrices," Arkiv för Matematik, vol. 50, no. 2, pp. 259-267, 2012.

[13] Y. Li and H. Du, "The intersection of essential approximate point spectra of operator matrices," Journal of Mathematical Analysis and Applications, vol. 323, no. 2, pp. 1171-1183, 2006.

[14] E. H. Zerouali and H. Zguitti, "Perturbation of spectra of operator matrices and local spectral theory," Journal of Mathematical Analysis and Applications, vol. 324, no. 2, pp. 992-1005, 2006.

[15] S. F. Zhang, Z. Y. Wu, and H. J. Zhong, "Continuous spectrum, point spectrum and residual spectrum of operator matrices," Linear Algebra and its Applications, vol. 433, no. 3, pp. 653-661, 2010.

[16] S. F. Zhang and H. J. Zhong, "A note on Browder spectrum of operator matrices," Journal of Mathematical Analysis and Applications, vol. 344, no. 2, pp. 927-931, 2008.

[17] S. F. Zhang, H. J. Zhong, and Q. F. Jiang, "Drazin spectrum of operator matrices on the Banach space," Linear Algebra and its Applications, vol. 429, no. 8-9, pp. 2067-2075, 2008.

[18] S. F. Zhang, H. J. Zhong, and J. D. Wu, "Spectra of $2 \times 2$ uppertriangular operator matrices," Acta Mathematica Sinica, vol. 54, no. 1, pp. 41-60, 2011 (Chinese).

[19] S. F. Zhang, H. J. Zhong, and J. D. Wu, "Fredholm perturbation of spectra of $2 \times 2$-upper triangular matrices," Acta Mathematica Sinica, vol. 54, no. 4, pp. 581-590, 2011 (Chinese).

[20] S. F. Zhang and J. D. Wu, "Samuel multiplicities and Browder spectrum of operator matrices," Operators and Matrices, vol. 6, no. 1, pp. 169-179, 2012. 
[21] Y. N. Zhang, H. J. Zhong, and L. Q. Lin, "Browder spectra and essential spectra of operator matrices," Acta Mathematica Sinica (English Series), vol. 24, no. 6, pp. 947-954, 2008.

[22] S. F. Zhang and Z. Q. Wu, "Characterizations of perturbations of spectra of $2 \times 2$ upper triangular operator matrices," Journal of Mathematical Analysis and Applications, vol. 392, no. 2, pp. 103-110, 2012.

[23] H. Zguitti, "On the Drazin inverse for upper triangular operator matrices," Bulletin of Mathematical Analysis and Applications, vol. 2, no. 2, pp. 27-33, 2010.

[24] X. Fang, "Samuel multiplicity and the structure of semiFredholm operators," Advances in Mathematics, vol. 186, no. 2, pp. 411-437, 2004.

[25] P. A. Fillmore and J. P. Williams, “On operator ranges," Advances in Mathematics, vol. 7, pp. 254-281, 1971. 


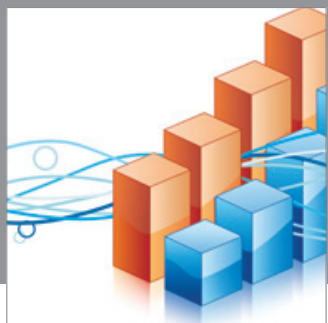

Advances in

Operations Research

mansans

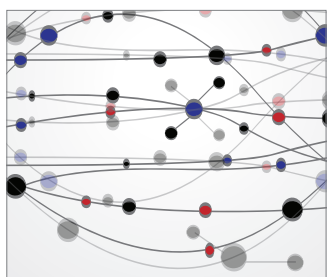

The Scientific World Journal
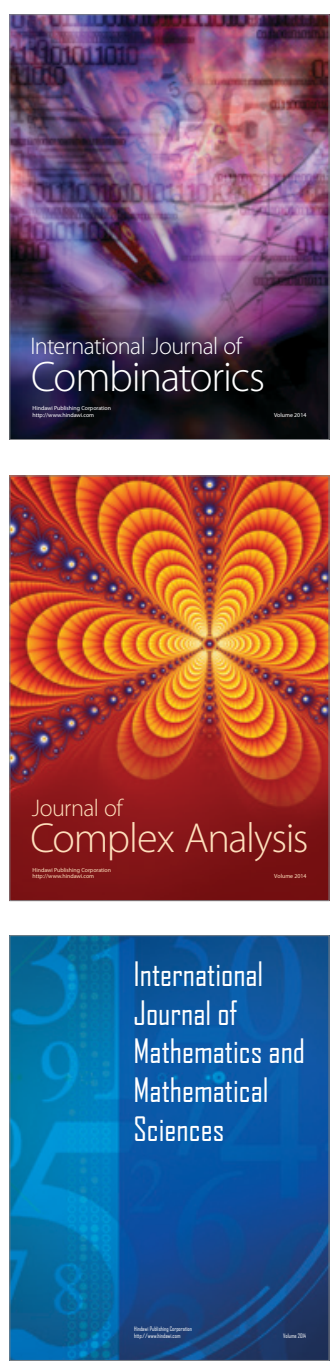
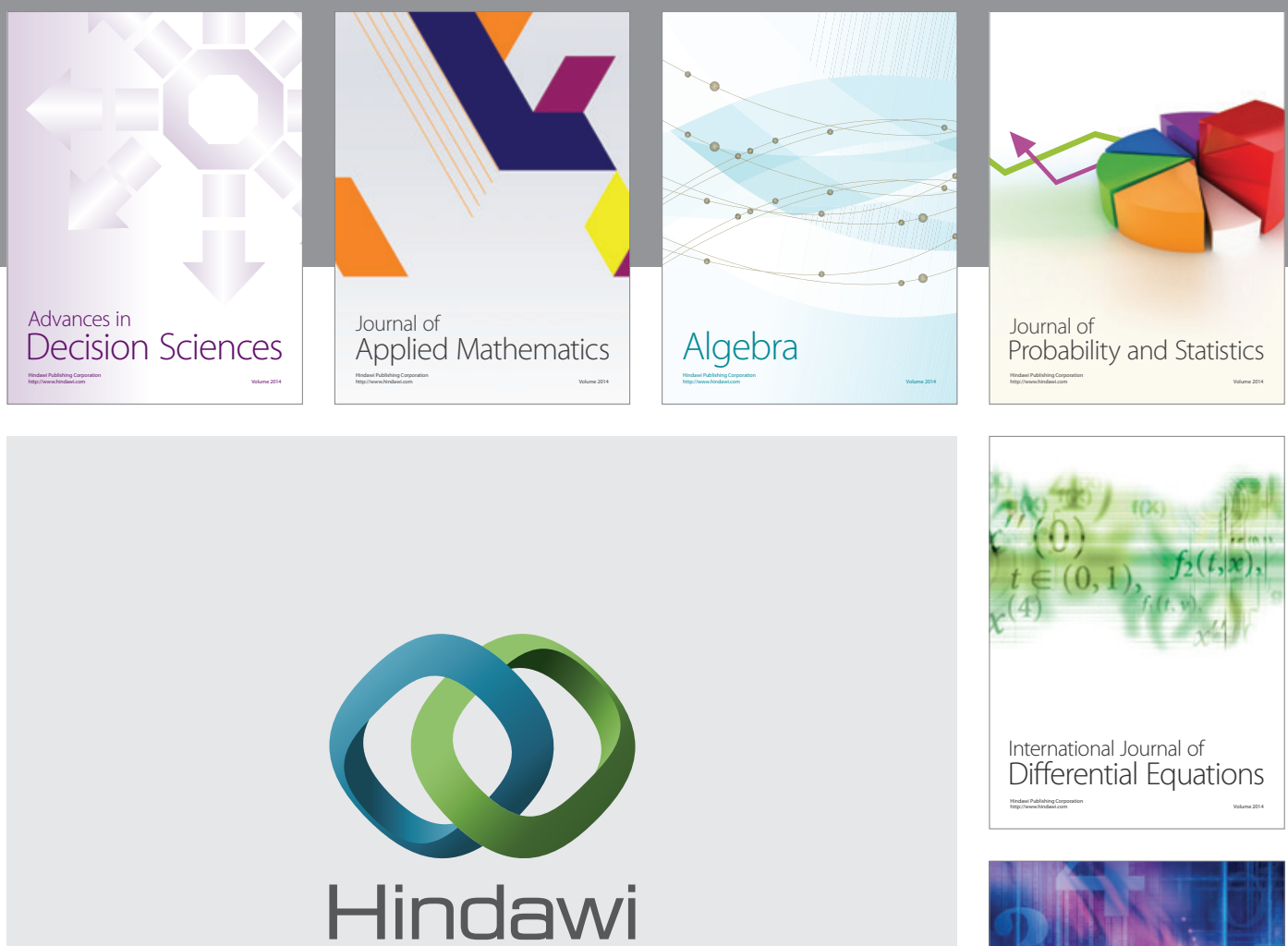

Submit your manuscripts at http://www.hindawi.com
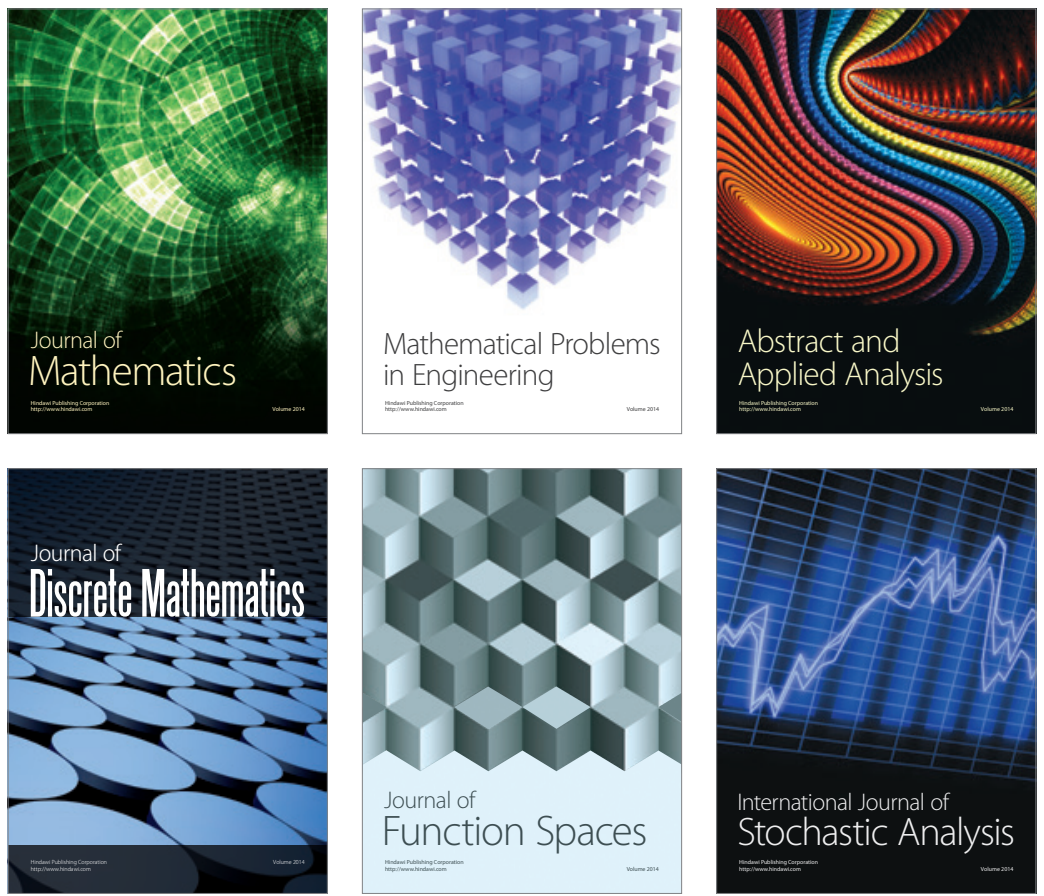

Journal of

Function Spaces

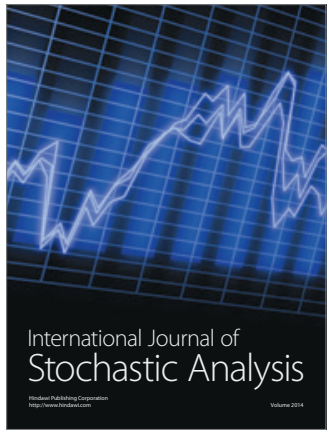

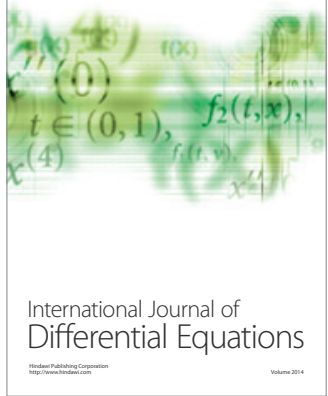
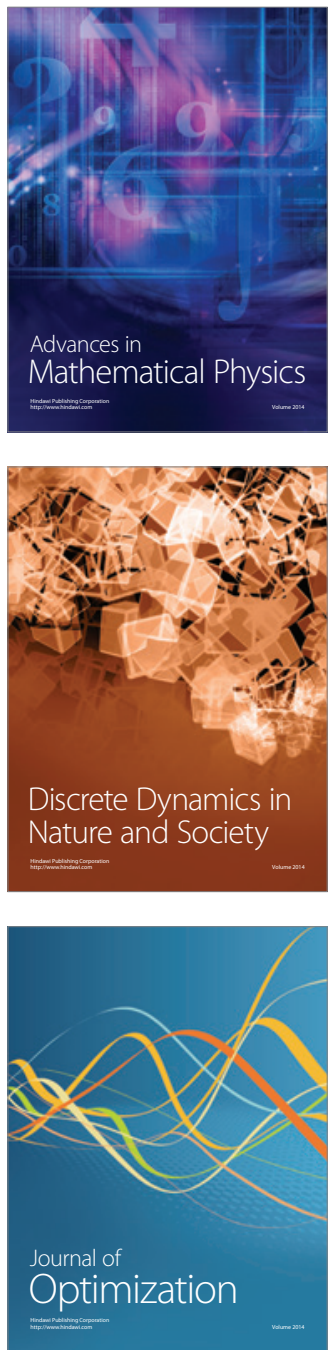